\title{
Evolving
}

\section{Efficacy and safety of right and left atrial ablations on the beating heart with irrigated bipolar radiofrequency energy: A long-term animal study}

Spencer J. Melby, MD, ${ }^{a}$ Sydney L. Gaynor, MD, ${ }^{a}$ Jordon G. Lubahn, BS, ${ }^{\text {a }}$ Anson M. Lee, MD, ${ }^{a}$ Paymon Rahgozar, BS, Shelton D. Caruthers, PhD, ${ }^{\mathrm{b}}$ Todd A. Williams, RT, ${ }^{\mathrm{b}}$ Richard B. Schuessler, PhD, and Ralph J. Damiano, Jr, MD ${ }^{\mathrm{a}}$

From the Division of Cardiothoracic Surgery, Department of Surgery, ${ }^{\mathrm{a}}$ and the Division of Cardiology, Department of Internal Medicine, ${ }^{\mathrm{b}}$ Washington University School of Medicine, St Louis, Mo.

Supported by a research grant from Medtronic Inc, and by National Institutes of Health grants 5R01 HL32257, F32 HL078136-01, and R44 HL67535. R.J.D., $\mathrm{Jr}$, has a consultant relationship with Medtronic Inc. S.D.C. reports equity ownership in Royal Philips Electronics; he is an employee of Philips Medical Systems.

Received for publication July 19, 2005; revisions received Feb 7, 2006; accepted for publication May 17, 2006.

Address for reprints: Ralph J. Damiano, Jr, MD, Chief, Cardiac Surgery, Washington University School of Medicine, $660 \mathrm{~S}$ Euclid Ave, Box 8234, St Louis, MO 63110 (E-mail: damianor@wustl.edu).

J Thorac Cardiovasc Surg 2006;132:853-60 $0022-5223 / \$ 32.00$

Copyright (C) 2006 by The American Association for Thoracic Surgery

doi:10.1016/j.jtcvs.2006.05.048
Objective: The Cox maze procedure is the most effective surgical treatment for atrial fibrillation; however, its complexity has limited its clinical utility. The purpose of this study was to simplify the procedure by using an irrigated bipolar radiofrequency ablation device on the beating heart without cardiopulmonary bypass.

Methods: Six domestic pigs underwent median sternotomy. The pulmonary veins were circumferentially ablated. Electrical isolation was confirmed by pacing. Eight lesions were performed epicardially, and three lesions were performed through purse-string sutures with one of the jaws of the device introduced into the right atrium. After 30 days, magnetic resonance imaging was performed to assess atrial function, pulmonary vein anatomy, and coronary artery patency. Cholinergic stimulation and burst pacing were administered to induce atrial fibrillation. Histologic assessment of the heart was performed after the animal was killed.

Results: A modified Cox maze procedure was successfully performed with the irrigated bipolar radiofrequency device with no deaths. In every instance, the pulmonary veins were electrically isolated. Cholinergic stimulation with burst pacing failed to produce atrial fibrillation. Imaging studies revealed tricuspid regurgitation without evidence of pulmonary vein stenosis, coronary artery stenosis, or intra-atrial thrombus. Total atrial ejection fraction was $16.9 \% \pm 7.5 \%$, a significant reduction. Histologically, $99 \%$ of the lesions were transmural, and there was no evidence of coronary sinus injury.

Conclusion: Lesions on both the right and left atria can be created successfully on the beating heart with irrigated bipolar radiofrequency. The great majority of lesions with this device were transmural. This device should not be used on valvular tissue.

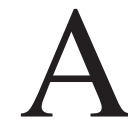

trial fibrillation (AF) is the most common of all sustained arrhythmias, with incidences in the United States as great as $1 \%$ in the general population and 9\% among people older than 80 years. ${ }^{1}$ It is associated with significant morbidity as a result of compromised hemodynamics, tachycardia-induced cardiomyopathy, and increased vulnerability to thromboembolism. This gives patients with AF a 5-fold risk of stroke, especially as age increases. ${ }^{2}$ Methods used in the treatment of AF include pharmacologic therapy, cardioversion, transvenous ablation, and surgery. 

Abbreviations and Acronyms
$\mathrm{AF}=$ atrial fibrillation
$\mathrm{CPB}=$ cardiopulmonary bypass
$\mathrm{IBRF}=$ irrigated bipolar radiofrequency
IVC = inferior vena cava
$\mathrm{PV}=$ pulmonary vein
$\mathrm{RF} \quad=$ radiofrequency

The Cox maze procedure is the most successful treatment for AF, with a long-term success rate of at least $90 \%$ and virtual elimination of late stroke. ${ }^{3-5}$ The procedure consists of a series of mazelike incisions or ablations on both atria. These are designed to create conduction block and prevent the macroreentrant circuits throughout the atria considered to be responsible for AF. ${ }^{5,6}$ After the first two iterations of the Cox maze procedure, the Cox maze III was first performed in 1988 and became the criterion standard for the surgical treatment of AF for more than a decade. ${ }^{3,7}$

Recently, modifications of the Cox maze procedure have been made that use various energy sources to create linear lines of ablation that replace the cut-and-sew lesions of the traditional procedure. ${ }^{8-10}$ These new technologies hold the promise of a less technically difficult way to perform the atrial lesions. The long-term efficacy of the various modifications to the Cox maze procedure are as yet unproven; however, simplification of the Cox maze III procedure by allowing its completion on a beating heart without the use of cardiopulmonary bypass (CPB), while preserving its efficacy and safety, may lead to wider application of this operation.

In this study, a modification of the Cox maze lesion set was created on the beating heart with an irrigated bipolar radiofrequency (IBRF) device. IBRF has been shown to effectively produce transmural lesions ex vivo. ${ }^{11}$ This device relies on saline solution as a conductor as alternating current is delivered between two approximated electrodes embedded into the jaws of a clamp. This allows focused delivery of energy that minimizes lesion width and reduces the risk of collateral damage to vital structures. An advantage of this technology is that by measuring real-time tissue impedance between the two electrodes it provides an indication of lesion transmurality. Dry bipolar radiofrequency (RF) energy has been shown in previous work to create discrete, transmural atrial lesions within 10 seconds. ${ }^{12,13}$

The purpose of this long-term animal study was to examine the feasibility and safety of using IBRF to perform left and right atrial ablations on the beating heart and to examine the effect of this energy on the atrial tissue, coronary arteries, and pulmonary veins (PVs) at 1 month.

\section{Materials and Methods}

\section{Experimental Protocol}

Six adult domestic pigs weighing 40 to $50 \mathrm{~kg}$ were used in this study. All animals received humane care in compliance with the "Guide for the Care and Use of Laboratory Animals" (http://www.nap.edu/catalog/5140.html).

Each animal underwent right and left atrial lesions on the beating heart. The animals were premedicated, intubated, and anesthetized with $2 \%$ to $4 \%$ isoflurane and monitored continuously during the procedure. The heart was exposed through a median sternotomy. The left and right PVs and the inferior vena cava (IVC) were isolated with umbilical tapes. The heart was paced from the right atrial appendage, the right atrial body, and the left and right PVs to establish pacing thresholds. The animals were given intravenous heparin $(350 \mathrm{U} / \mathrm{kg})$ to maintain an activated clotting time longer than 250 seconds. Intravenous bretylium tosylate (INN bretylium tosilate) was administered to prevent arrhythmias with manipulation of the heart.

An IBRF surgical ablation device, Cardioblate BP Surgical Ablation System (Medtronic Inc, Minneapolis, Minn) was used to create the lesion set. The device consists of a hand piece, embedded bipolar electrodes in a clamp, and RF generator. RF energy was delivered to the two electrodes located in the opposing jaws of the clamp, which were mounted on an articulating head. The head could be angulated through $90^{\circ}$ and could also be rotated through $300^{\circ}$ at its articulating surface. Additionally, the jaws of the clamp were malleable, providing another degree of flexibility to the device that allowed the device to be shaped to match the target tissue. During RF ablation the contact tissue surface was constantly irrigated with saline solution, which acted as a conductor for the delivered energy. The generator delivered RF energy while an online system in real time monitored tissue impedance, current, voltage delivered, and duration of ablation. The devices used in this study operated on the principle that tissue is fully ablated when impedance reaches a stable plateau. Initially, moderate power was applied to the tissue. Impedance was measured continuously, and the derivative of impedance was calculated every $200 \mathrm{~ms}$. When impedance achieved a stable plateau, the algorithm logic determined that maximum ablation at this power level was complete. The power was then increased by a step function of $5 \mathrm{~W}$. If the plateau in impedance was not sustained, then the algorithm determined that transmurality had not been achieved, and ablation continued until another plateau in impedance was detected. This process was repeated until an impedance plateau was sustained after an increase in power. At this point, the microprocessor determined that transmurality had been achieved, and the generator provided a signal to the user.

\section{Surgical Procedure}

The modified Cox maze procedure included nearly all the right and left atrial lesions of the traditional procedure (Figure 1). All lesions were performed with the IBRF device. In performing this procedure without $\mathrm{CPB}$, it was necessary to make the following modifications of the classic Cox maze III procedure: (1) the right and left PVs were isolated separately, rather than as one large island; (2) a connecting lesion was performed between the right and left PVs; (3) the transseptal incision, which functions principally to aid 


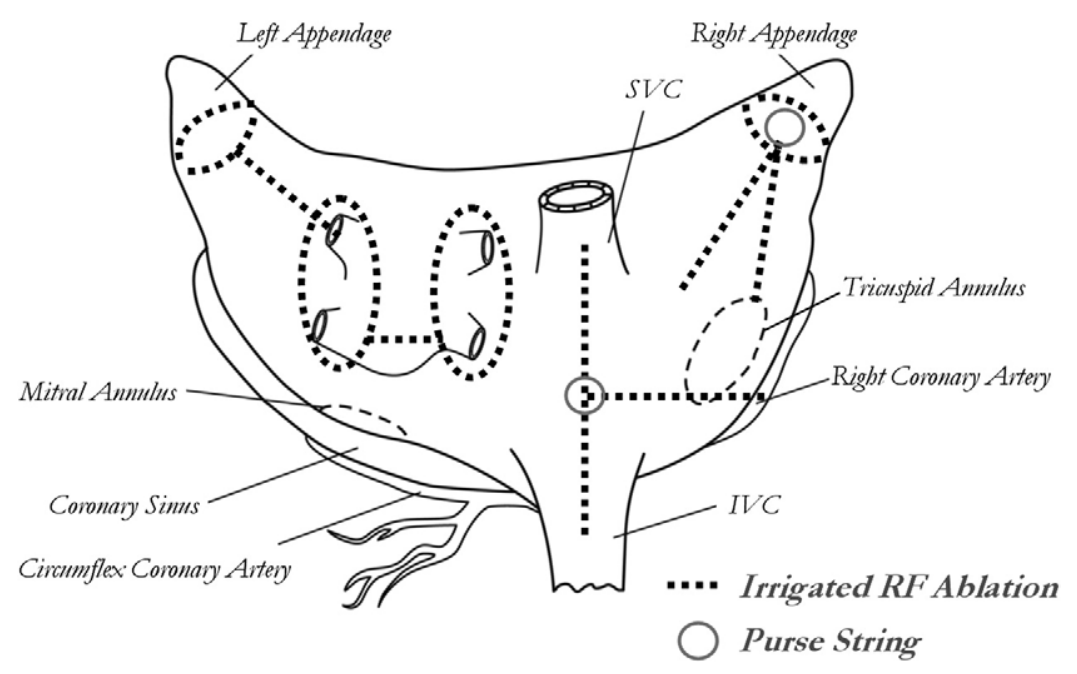

Figure 1. Modified Cox maze lesion set made with IBRF device. SVC, Superior vena cava.

in exposure of the posterior left atrium, was omitted; (4) the lesion connecting the PVs to the mitral annulus was also omitted.

The heart was manipulated with the aid of a suction retractor (AXIUS Xpose 3; Guidant Corp, Santa Clara, Calif). The IBRF device was passed around the right PVs, and a cuff of surrounding atrial tissue was clamped and ablated on each side; the same was done around the left PVs. Electrical isolation was confirmed by pacing the PVs distal to the lesions at a stimulus strength of 20 $\mathrm{mA}$. The IBRF device was used to circumferentially ablate the left atrial appendage on the epicardial surface. The device was then used to grasp a line of atrial tissue along the anterior aspect of the left atrial appendage, with the tip directed onto the left superior PV to create the connecting lesion from the left atrial appendage to the left PV. This was done entirely on the epicardial surface and resulted in two approximately parallel lesions.

The heart was repositioned to expose the intra-atrial groove. The IBRF device was placed epicardially posterior to the IVC, with tissue from the right inferior PV to the left inferior PV within the jaws of the clamp to create a connecting lesion between the inferior aspect of the PVs. The interposing tissue was ablated, completing the connecting lesion between the left and right PVs. Ablation in this manner resulted in two closely approximated lesions.

The IBRF device was introduced through a purse-string suture at the tip of the right atrial appendage and positioned to create the right atrial free wall lesion. The device was then placed across the tricuspid valve, crossing the atrioventricular groove, and the tissue, including the right coronary artery, was ablated. The right atrial appendage was circumferentially ablated. A purse-string suture was placed midway between the confluence of the superior vena cava and IVC. The IBRF device was introduced through this purse-string suture, and superior vena cava and IVC lesions were created. The snare was relaxed, and the device was carefully repositioned into the right ventricle. This lesion incorporated the tricuspid valve, atrioventricular groove, and right coronary artery. The IBRF device was removed and hemostasis secured. This completed the modified Cox maze lesion set (Figure 1). The pericardium was closed and the sternum reapproximated.

\section{Postoperative Data Collection}

Postoperatively, the animals were closely monitored for 48 hours and survived for 30 days. Each animal received $62.5 \mathrm{mg}$ aspirin a day, commencing on the first postoperative day. No antiarrhythmic drugs were used during the recovery period.

At 1 month, each animal underwent a magnetic resonance imaging (MRI) scan to assess the following: (1) atrial function, (2) the mitral and tricuspid valves, (3) PV anatomy and flow, and (4) coronary artery anatomy and flow. This was followed immediately

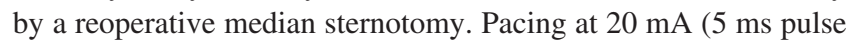
duration) was used to document chronic electrical isolation of the left and right PVs. Neostigmine bromide $(2-3 \mathrm{mg} / 50 \mathrm{~kg}$ ) was administered intravenously. Burst pacing was performed from the right atrial appendage and left and right atrial bodies at a cycle length of 30 to $100 \mathrm{~ms}$ continuously for 30 seconds in an attempt to induce AF. ${ }^{14,15}$ In a control group of healthy animals, 8 pigs underwent a median sternotomy and pericardiotomy. Before antiarrhythmic medication was given, 8 of 8 animals (100\%) went into AF spontaneously on opening of the pericardium $(n=2)$ or by induction with burst pacing $(n=6)$. Animals sustained AF for a range of 1 to 8 minutes and then converted back to normal sinus rhythm spontaneously $(\mathrm{n}=3)$ or by administration of bretylium tosylate $(\mathrm{n}=5)$.

\section{MRI Protocol}

The animals were anesthetized and placed supine in a clinical 1.5-T MRI scanner (NT Intera CV; Philips Medical Systems, Best, The Netherlands) and fitted with a 5-element dedicated cardiac surface coil for image reception. The imaging protocol consisted of multiple anatomic and functional cine images and velocityencoded images. All images were synchronized to the cardiac rhythm with a 4-lead vectorcardiogram system. The cine images, triggered to each R-wave peak, were based on a steady-state balanced fast field echo or balanced turbo field echo technique with 15 phases per cardiac cycle spaced 20 to $25 \mathrm{~ms}$ apart. The turbo field echo technique sequence was superior in detecting volumes and did not truncate data at the end of the cardiac cycle; 
it was therefore implemented in the final animals. The cine MRI views consisted of the following: (a) horizontal long axis, (b) vertical long axis, (c) short-axis views of 20 planes, $9 \mathrm{~mm}$ thick, and (d) left ventricular outflow track. The reconstructed pixel size was approximately $1.5 \times 1.2 \mathrm{~mm}$. Transtricuspid blood flow was assessed with velocity-encoded MRI. ${ }^{16}$

\section{MRI Data Analysis}

Analysis of the acquired images was manually performed off-line using a commercial software package (Easy Vision, Philips Medical System, Best, The Netherlands). The volume of the left atrium was assessed by planimetry at each time point of the cardiac cycle. The area of the left atrial blood pool was identified in each short-axis slice. The image was hand-traced using a cursor and the volumes of the left atrium were calculated and summed over all slices to give the estimated total volume of the left atrium at each time point of the cardiac cycle, including end systole and end diastole. Atrial ejection fraction was then defined as $[(\mathrm{EDV}-\mathrm{ESV})] / \mathrm{EDV} \times 100 \%$, where $E D V$ is end-diastolic volume and ESV is end-systolic volume. Analysis of quantitative flow across the tricuspid valve was performed with velocity-encoded images. MRI was used to assess anatomic and physiologic flow patterns in the pulmonary venous system.

\section{Coronary Artery Anatomy and Patency}

The technique of Botnar and colleagues, ${ }^{17}$ which uses a navigatorgated free-breathing T2-prepared turbo field echo acquisition, was used to assess coronary artery anatomy and patency.

\section{Histologic and Microscopic Analyses}

The animals were humanely killed, and the hearts were removed en bloc and examined grossly for any evidence of intra-atrial thrombus formation, PV stenosis, or thrombosis. The hearts were immediately placed in 1\% 2,3,5-triphenyl-tetrazolium chloride solution and incubated at room temperature for 45 minutes. Each IBRF lesion was sectioned three times when possible, $5.0 \mathrm{~mm}$ apart, perpendicular to the long axis of the ablation line. The sections were then fixed in formalin, molded in paraffin, sectioned, and stained with hematoxylineosin and Gomori trichrome stains. The sections were microscopically examined to assess transmurality of the lesions and the effects of IBRF energy on the tricuspid valve.

\section{Results}

\section{Operative Results}

All animals survived the operative procedure. The mean time to complete the ablation procedure was $46 \pm 21$ minutes (range 23-80 minutes). There was no evidence of cardiac arrhythmia, and all animals remained in sinus rhythm during the operation and up to when they were killed. There were no signs of gross neurologic dysfunction in any of the animals after the procedure.

\section{RF Lesions}

The beating heart lesion set consisted of 11 IBRF ablations (Figure 1). Two lesions traversed the tricuspid valve. The right coronary was deliberately crossed with the IBRF device. Acutely, the PVs were electrically isolated with a

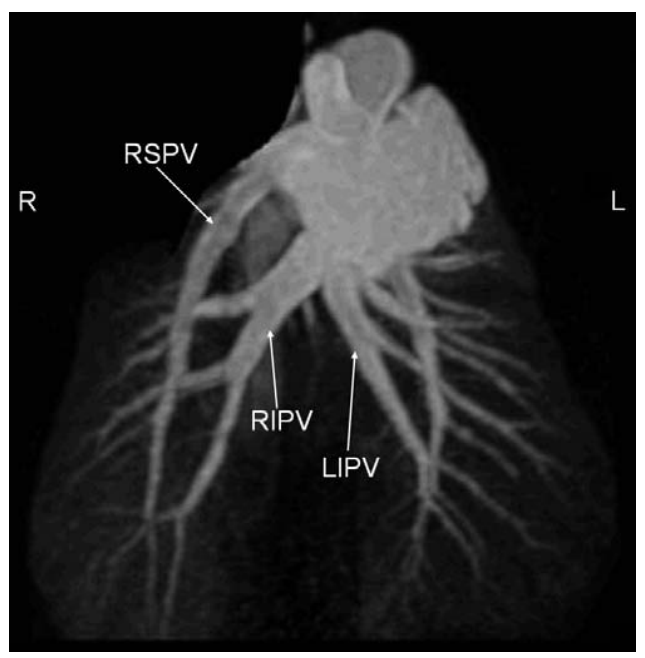

Figure 2. Representative MRI indicating PV patency (arrows) at 1 month after ablation with IBRF device. RSPV, Right superior PV, $R I P V$, right inferior PV, LIPV, left inferior PV.

single ablation in all instances; this was documented by failure of the heart to capture while pacing from the PVs at $20 \mathrm{~mA}$. All lesions were created with a single application of the IBRF device. An integrated tissue impedance monitor located within the device, which has been previously described by Hamner and coworkers, ${ }^{11}$ determined ablation times. The maximum power delivered during each ablation was $28 \pm 4 \mathrm{~W}$ (range 18-39 W).

\section{Cardiac Function Data}

Functional cardiac MRI analysis was available for 5 of the 6 animals after surgery; these results were compared with MRIs from a pool of healthy animals $(n=11)$. The total left atrial ejection fraction seen in the healthy animals was $32.9 \% \pm 6.5 \%$. In the study group, the postoperative total left atrial ejection fraction was $16.9 \% \pm 7.5 \%(P<.01)$. In comparison, the active left atrial ejection fraction (the volume corresponding to active atrial contraction) assessed in 5 healthy animals was $19.1 \% \pm 6.8 \%$; in the 5 evaluated IBRF-treated animals, the active atrial ejection fraction was $8.5 \% \pm 2.2 \%(P<.03)$. Atrial contractility, as documented by the presence of an A wave, was present postoperatively in all 5 treated animals.

There was no evidence of PV stenosis in any animal (Figure 2). Quantitative flow studies to evaluate for regurgitation were available for only 4 of 6 animals after surgery. Tricuspid regurgitation was seen in all 4 animals studied $(100 \%)$, with an average regurgitant fraction of $12.7 \% \pm$ $7.3 \%$; 2 animals showed moderate tricuspid regurgitation and 2 showed severe regurgitation. 

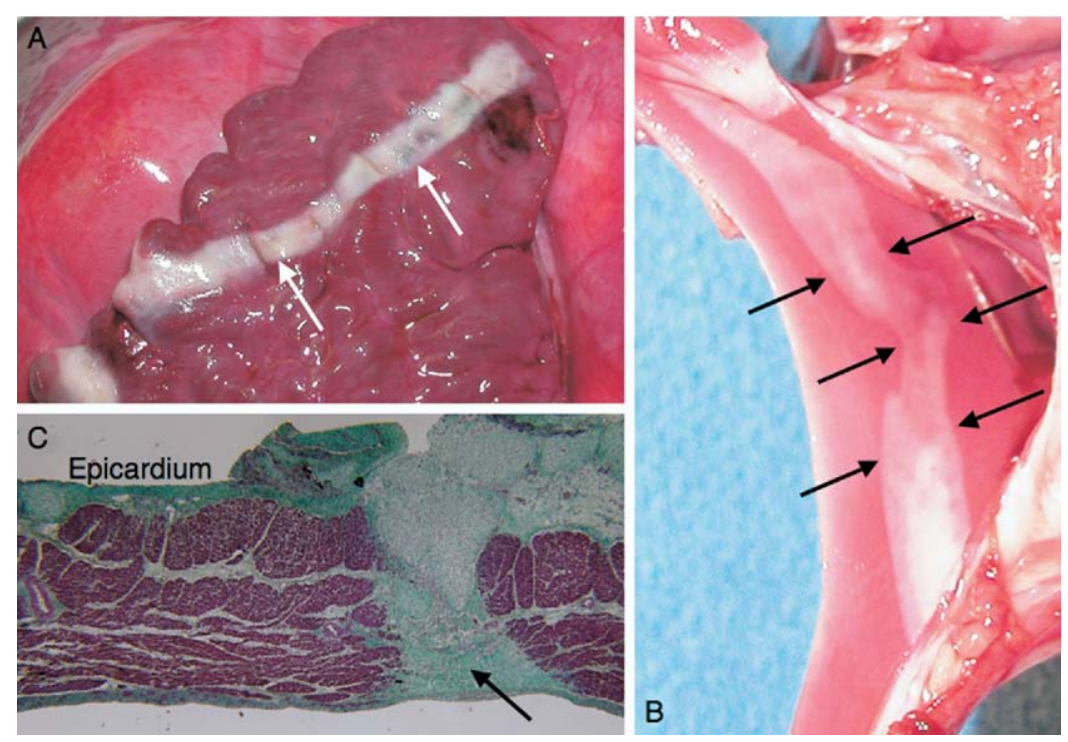

Figure 3. Ablations made with IBRF device (arrows). A, Right atrial appendage acute ablation. B, Demonstration of left PV chronic ablation after 1 month. C, Photomicrograph of atrial tissue 1 month after ablation by IBRF device (Gomori trichrome stain, original magnification $20 \times$ ).

MRI evaluation did not detect any coronary artery stenosis or thrombosis in the areas ablated. The right coronary artery remained patent in each animal.

\section{Chronic Pacing}

At 30 days, pacing demonstrated that the right atrial appendage and the right and left PVs were isolated from the body of the atria in every animal. During normal sinus rhythm or atrial pacing from the body of the atria, the right atrial appendage was electrically silent. Attempts to induce AF from both left and right atria with burst pacing coupled with intravenous neostigmine were unsuccessful in $100 \%$ of the animals (6/6). In comparison, AF was induced in $100 \%$ of healthy animals $(n=8)$. Neostigmine was used because it increases cholinergic tone and shortens the effective refractory period, thus facilitating reentry and AF. This has also been documented in other animal models. ${ }^{14,15}$

\section{Histology}

On gross inspection of the heart, there was no evidence of intra-atrial thrombus formation or stricture of the PVs. At necropsy, the hearts were placed in 1\% 2,3,5-triphenyltetrazolium chloride solution and examined. The atrial lesions appeared pale, discrete, and linear (Figure 3).

Three representative sections were taken from each of the 11 ablations when possible (Figure 1). A total of 126 sections were examined from the 6 animals. Transverse and linear histologic sections of ablative tissue demonstrated continuous, full-thickness replacement of myocardial fibers with septa of maturing fibrous connective tissue.

Microscopic examination of the atrial samples stained with Gomori trichrome revealed that 125 of the 126 lesions were transmural (99.2\%; Table 1). Average lesion width of a representative sample of the ablated tissues $(n=88)$ was
$3.4 \pm 1.1 \mathrm{~mm}$ (range 1.3-6.6 mm). The single nontransmural lesion was located on the left atrial appendage. If the appendage lesion was eliminated from analysis, because this lesion is not performed clinically and the left atrial appendage is typically amputated, then $100 \%$ (100/100) of the lesions were transmural. Lesions were well connected and overlapped, including the PV connecting lesions.

Histologic examination of the ablated tricuspid valve tissue revealed a discrete ablation line with no perforation of the leaflets. The subvalvular apparatus remained intact. There was no valvular thrombus.

\section{Discussion}

The Cox maze procedure has proven to be a lasting and highly effective treatment for AF. ${ }^{3-5}$ The technical difficulty of the procedure and the associated risks of placing patients on $\mathrm{CPB}$ for prolonged periods, however, has spurred interest in less invasive approaches to the surgical treatment of AF. ${ }^{9-13}$ The goal of any new operation is to preserve the efficacy of the Cox maze procedure and at the same time

TABLE 1. Lesion transmurality

\begin{tabular}{lccc}
\hline Lesion site & $\begin{array}{c}\text { Total } \\
\text { (No.) }\end{array}$ & $\begin{array}{c}\text { Transmural } \\
\text { lesions } \\
\text { (No.) }\end{array}$ & $\begin{array}{c}\text { Width } \\
\text { (mm, mean } \pm \text { SD) }\end{array}$ \\
\hline Atrium & 67 & $66(99 \%)$ & $3.3 \pm 0.9$ \\
PV & 23 & $23(100 \%)$ & $4.2 \pm 0.1$ \\
Vena cava & 19 & $19(100 \%)$ & $2.9 \pm 2.0$ \\
Transtricuspid valve & 17 & $17(100 \%)$ & $3.9 \pm 0.8$ \\
Total & 126 & $125(99 \%)$ & $3.4 \pm 1.1$ \\
\hline
\end{tabular}

All lesions were made with the IBRF device. Histologic sections were taken from all 6 animals. 
decrease the morbidity. Thus the purpose of this study was to evaluate an approach on the beating heart that would not require $\mathrm{CPB}$.

To facilitate the procedure, it was decided to use IBRF ablation to replace the surgical incisions. The use of ablation lines in lieu of surgical incisions has several advantages. First of all, clamping can be performed without CPB and does not require an arrested heart, as does the traditional cut-and-sew approach. The use of a clamp as opposed to a complicated set of incisions simplifies the procedure and should make it more accessible to cardiac surgeons. Second, ablation devices are faster than the traditional cut-and-sew method. Furthermore, the lack of long suture lines may reduce the risk of postoperative bleeding.

IBRF technology is of particular interest because in this technology, saline irrigation of the electrodes maintains a low electrode-surface interface temperature, which allows the thermal energy of the device to penetrate deeper in the tissue of interest. Irrigated RF devices have been shown to create deeper lesions than pulsed and conventional RF and microwave devices. ${ }^{18,19}$ To avoid the risk of damaging collateral tissues associated with unipolar RF devices, the use of saline irrigation has been incorporated into bipolar RF devices. Additionally, bipolar RF devices have the capability to provide immediate feedback on lesion transmurality, giving indication that complete conduction block has been created. ${ }^{12}$ In early studies, the use of an IBRF clamp was highly effective at creating transmural lesions on ex vivo porcine atria. ${ }^{11}$

It was our goal in this study to examine the feasibility of creating both right and left atrial lesions with an IBRF device. In the traditional procedure, both the right and left atrial appendages are resected. However, increasing evidence suggests that preservation of the atrial appendages maintains production of atrial natriuretic peptide and may reduce the incidence of postoperative fluid retention. ${ }^{20,21} \mathrm{In}$ light of this, the left and right atrial appendages were ablated but not excised in this experiment. This allowed us to document electrical isolation and to generate a line of conduction block associated with a traditional Cox maze procedure. In the clinical situation at our institution, the right atrial appendage is preserved but the left atrial appendage is always amputated, to decrease the risk of postoperative stroke. The septal lesion was deliberately eliminated in this study because (1) it is not accessible through the epicardial approach, and (2) traditionally, this lesion has been performed only for exposure and is not a part of our current version of the Cox maze procedure. ${ }^{9}$ There may be a subset of patients in whom this lesion is important, however, and further clinical investigation is needed to determine whether this lesion carries significance. Recent evidence has shown that ectopic foci located in the PVs are responsible for the initiation of $\mathrm{AF}$ in most cases. ${ }^{22}$ Early data also suggests that PV ablation may be a more effective treatment of AF than antiarrhythmic drug therapy alone. ${ }^{23}$ In light of this evidence and in an attempt simplify the procedure, the mitral annular lesion performed in the traditional Cox maze lesion set was omitted in this study. This is a difficult lesion to perform on the beating heart with a bipolar RF device. The omission of the lesion connecting the PV isolation to the mitral annulus, however, has the potential for creating postoperative atrial flutter. Although none of the animals showed this complication, postoperative atrial flutter has been seen in human beings where this lesion was missing or incomplete. ${ }^{24}$ Although the connecting lesions between the PVs were difficult in the porcine model because they required dissection of the posterior heart space, this technique would not be possible in the human heart because of the difficulty of folding the posterior atrial tissue within the clamp. A connecting lesion likely would require institution of CPB and introduction of the clamp into the left atrium.

Our results have demonstrated that IBRF energy can be used on the beating heart to create transmural lesions that replace many of the standard lesions of the Cox maze procedure. AF could not be induced in any animal with cholinergic stimulation. These lesions resulted in chronic transmural conduction block, as documented by pacing. On histologic examination, virtually all the lesions were transmural. If the left atrial appendage lesion was eliminated from analysis, because it is not performed clinically, all the lesions $(100 \%)$ were transmural. Thus the transmurality algorithm is highly reliable in this animal model.

To evaluate the safety of this approach, the animals underwent postoperative MRI evaluation to assess cardiac anatomy and atrial function. Cardiac MRI was chosen instead of echocardiography because of its superior edge detection and the ability to delineate structures as small as 1 to $2 \mathrm{~mm} .{ }^{25}$ There was no evidence of PV stenosis with IBRF ablation. This is in contrast to the late PV stenosis seen with catheter-based, unipolar RF ablation. ${ }^{23,26,27}$

Atrial ejection fraction was decreased after this procedure. Of the 5 animals examined, however, all had atrial contractility, as documented by the presence of an A wave. It should be noted that surgery was done on normal pig atria with normal sinus rhythm. Thus in a normal, healthy atrium, the modified Cox maze procedure completed by the IBRF device will likely lead to a decrease in atrial ejection fraction. Patients undergoing the Cox maze procedure, however, have diseased, nonfunctioning atria. Therefore these results pertaining to a loss of atrial ejection fraction may not correlate with clinical experience. Nonetheless, further research is needed to better understand the relationship between left atrial ablation and atrial function to maximize postoperative left atrial emptying.

The chronic effects of IBRF ablation on the right coronary artery and the tricuspid valve were evaluated. Surpris- 
ingly, MRI angiography documented no evidence of coronary artery stenosis or thrombosis after the procedure. This does not define safety, however, and a longer-term study including focused histologic examination would be needed to rule out the induction of intimal hyperplasia and eventual coronary artery stenosis. Until this has been demonstrated, clinical ablation of coronary arteries should be avoided. On MRI, the animals had significant postoperative tricuspid regurgitation. The tricuspid regurgitation was likely a direct consequence of ablation across valvular tissue with the IBRF device. This finding highlights the importance of avoiding any ablation of the valve leaflets when using this device.

In summary, this study demonstrated the feasibility of a beating-heart operation that included many of the standard lesions of the Cox maze procedure. IBRF ablation was effective in creating transmural lesions according to both electrophysiologic and histologic evaluation. This energy source was safe and did not result in injury to the PVs. Clinical trials of this technology on the beating heart are warranted and may lead to a more minimally invasive Cox maze procedure.

\section{Study Limitations}

Because of the inherent limitations of this study, these results must be cautiously applied to the clinical situation. First, there are significant differences between human anatomy and that of the porcine model. The larger PVs and significant fat in patients may make ablation more difficult. Moreover, this study was performed on normal porcine atria, and not on the diseased and often thickened human atria seen in patients with AF. Our clinical experience with $\mathrm{CPB}$, however, has similarly documented the efficacy of creating transmural lesions with bipolar RF technology. ${ }^{9,10}$

This experimental procedure involved using IBRF ablation across the right coronary artery. Although there was no evidence of thrombosis or stenosis found by MRI, the follow-up was at only 1 month and does not rule out the possibility of late injury. With both RF and microwave energy in the clinical setting, there have been reports of coronary artery damage. ${ }^{28-30}$ This procedure can be modified to avoid these structures by reflecting the atrioventricular groove fat pad or crossing the groove distal to the last circumflex marginal coronary artery. We emphasize that this study should not in any way be construed as evidence that ablating coronary arteries is safe; indeed, we strongly recommend against it.

Another limitation of this study is that the MRI studies to evaluate atrial function, the atrioventricular valves, the PVs, inducibility of AF, and coronary artery anatomy and flow were only done postoperatively. These data were compared with MRI data collected from healthy animals. The lack of preoperative MRI data on the study animals prevented the observation of changes in each animal induced by the procedure. There is also the possibility that the pericardiotomy itself (in a setting without ablations) might influence the changes seen in the postsurgical setting. To create this control group of animals is cost-prohibitive and will need to be addressed in future investigations.

Finally, a significant shortcoming for the clinical use of this procedure is the need to place the clamp inside the atrium. Although this likely can be performed with little difficulty on the right side of the heart, there would be a chance for both bleeding and air embolus on the left side. If a more expanded lesion set is found to be necessary, this could limit the utility of bipolar RF and may require the development of other parallel technology.

We thank Dr Samuel A. Wickline for his assistance in the interpretation of the MRI studies.

\section{References}

1. Go AS, Hylek EM, Phillips KA, Chang Y, Henault LE, Selby JV, et al. Prevalence of diagnosed atrial fibrillation in adults: national implications for rhythm management and stroke prevention: the AnTicoagulation and Risk Factors in Atrial Fibrillation (ATRIA) Study. JAMA. 2001;285:2370-5.

2. Wolf PA, Abbott RD, Kannel WB. Atrial fibrillation as an independent risk factor for stroke: the Framingham Study. Stroke. 1991;22:983-8.

3. Cox JL, Boineau JP, Schuessler RB, Jaquiss RD, Lappas DG. Modification of the maze procedure for atrial flutter and atrial fibrillation. I. Rationale and surgical results. J Thorac Cardiovasc Surg. 1995;110: 473-84.

4. Prasad SM, Maniar HS, Camillo CJ, Schuessler RB, Boineau JP, Sundt TM 3rd, et al. The Cox-Maze procedure for atrial fibrillation: longterm efficacy in patients undergoing lone versus concomitant procedures. J Thorac Cardiovasc Surg. 2003;126:1822-8.

5. Cox JL, Schuessler RB, D'Agostino HJ Jr, Stone CM, Chang BC, Cain $\mathrm{ME}$, et al. The surgical treatment of atrial fibrillation. III. Development of a definitive surgical procedure. J Thorac Cardiovasc Surg. 1991; 101:569-83

6. Cox JL, Canavan TE, Schuessler RB, Cain ME, Lindsay BD, Stone C, et al. The surgical treatment of atrial fibrillation. II. Intraoperative electrophysiologic mapping and description of the electrophysiologic basis of atrial flutter and atrial fibrillation. J Thorac Cardiovasc Surg. 1991;101:406-26.

7. Cox JL. The standard maze-III procedure. In: Cox JL, editor. Operative techniques in thoracic and cardiovascular surgery: a comparative atlas, vol 5, No 1. Orlando (FL): WB Saunders; 2000. p. 2-22.

8. Viola N, Williams MR, Oz MC, Ad N. The technology in use for the surgical ablation of atrial fibrillation. Semin Thorac Cardiovasc Surg. 2002;14:198-205.

9. Gaynor SL, Diodato MD, Prasad SM, Ishii Y, Schuessler RB, Bailey MS, et al. A prospective, single-center clinical trial of a modified Cox maze procedure with bipolar radiofrequency ablation. J Thorac Cardiovasc Surg. 2004;128:535-42.

10. Mokadam NA, McCarthy PM, Gillinov AM, Ryan WH, Moon MR, Mack MJ, et al. A prospective multicenter trial of bipolar radiofrequency ablation for atrial fibrillation: early results. Ann Thorac Surg. 2004;78:1665-70.

11. Hamner CE, Lutterman A, Potter DD, Sundt TM 3rd, Schaff HV, Francischelli D. Irrigated bipolar radiofrequency ablation with transmurality feedback for the surgical Cox-maze procedure. Heart Surg Forum. 2003;6:418-23.

12. Prasad SM, Maniar HS, Schuessler RB, Damiano RJ Jr. Chronic transmural atrial ablation by using bipolar radiofrequency energy on the beating heart. J Thorac Cardiovasc Surg. 2002;124:708-13. 
13. Prasad SM, Maniar HS, Diodato MD, Schuessler RB, Damiano RJ Jr. Physiological consequences of bipolar radiofrequency energy on the atria and pulmonary veins: a chronic animal study. Ann Thorac Surg. 2003;76:836-42.

14. Schuessler RB, Grayson TM, Bromberg BI, Cox JL, Boineau JP. Cholinergically mediated tachyarrhythmias induced by a single extrastimulus in the isolated canine right atrium. Circ Res. 1992;71:1254-67.

15. Geddes LA, Hinds M, Babbs CF, Tacker WA, Schoenlein WE, Elabbady $\mathrm{T}$, et al. Maintenance of atrial fibrillation in anesthetized and unanesthetized sheep using cholinergic drive. Pacing Clin Electrophysiol. 1996;19:165-75.

16. Nayler GL, Firimin DN, Longmore DB. Blood flow imaging by cine magnetic resonance. J Comput Assist Tomogr. 1986;10:715-22.

17. Botnar RM, Stuber M, Danias PG, Kissinger KV, Manning WJ. Improved coronary artery definition with T2-weighted, free-breathing, three-dimensional coronary MRA. Circulation. 1999;99:3139-48.

18. Erdogan A, Grumbrecht S, Neumann T, Neuzner J, Pitschner HF. Microwave, irrigated, pulsed, or conventional radiofrequency energy source: which energy source for which catheter ablation? Pacing Clin Electrophysiol. 2003;26:504-6.

19. Nakagawa H, Yamanashi WS, Pitha JV, Arruda M, Wang X, Ohtomo $\mathrm{K}$, et al. Comparison of in vivo tissue temperature profile and lesion geometry for radiofrequency ablation with a saline-irrigated electrode versus temperature control in a canine thigh muscle preparation. Circulation. 1995;91:2264-73.

20. Isobe F, Kumano H, Ishikawa T, Sasaki Y, Kinugasa S, Nagamachi K, et al. A new procedure for chronic atrial fibrillation: bilateral appendage-preserving maze procedure. Ann Thorac Surg. 2001;72:1473-8.

21. Yoshihara F, Nishikimi T, Sasako Y, Kobayashi J, Kosakai Y, Hattori $\mathrm{R}$, et al. Preservation of the right atrial appendage improves reduced plasma atrial natriuretic peptide levels after the maze procedure. J Thorac Cardiovasc Surg. 2000;119:790-4.
22. Haissaguerre M, Jais P, Shah DC, Takahashi A, Hocini M, Quiniou G, et al. Spontaneous initiation of atrial fibrillation by ectopic beats originating in the pulmonary veins. N Engl J Med. 1998;339:659-66.

23. Wazni OM, Marrouche NF, Martin DO, Verma A, Bhargava M, Saliba $\mathrm{W}$, et al. Radiofrequency ablation vs antiarrhythmic drugs as first-line treatment of symptomatic atrial fibrillation: a randomized trial. JAMA. 2005;293:2634-40.

24. Cox JL, Ad N. The importance of cryoablation of the coronary sinus during the maze procedure. Semin Thorac Cardiovasc Surg. 2000;12: 20-4.

25. Pujadas S, Reddy GP, Weber O, Lee JJ, Higgins CB. MR imaging assessment of cardiac function. J Magn Reson Imaging. 2004;19:78999.

26. Arentz T, Jander N, von Rosenthal J, Blum T, Furmaier R, Gornandt $\mathrm{L}$, et al. Incidence of pulmonary vein stenosis 2 years after radiofrequency catheter ablation of refractory atrial fibrillation. Eur Heart J. 2003;24;963-9.

27. Karch MR, Zrenner B, Deisenhofer I, Schreieck J, Ndrepepa G, Dong $\mathrm{J}$, et al. Freedom from atrial tachyarrhythmias after catheter ablation of atrial fibrillation: a randomized comparison between 2 current ablation strategies. Circulation. 2005;111:2875-80.

28. Fayad G, Modine T, Le Tourneau T, Decoene C, Azzaoui R, AlRuzzeh S, et al. Circumflex artery stenosis induced by intraoperative radiofrequency ablation. Ann Thorac Surg. 2003;76:1291-3.

29. Manasse E, Medici D, Ghiselli S, Ornaghi D, Gallotti R. Left main coronary arterial lesion after microwave epicardial ablation. Ann Thorac Surg. 2003;76:276-7.

30. Kottkamp H, Hindricks G, Autschbach R, Krauss B, Strasser B, Schirdewahn P, et al. Specific linear left atrial lesions in atrial fibrillation: intraoperative radiofrequency ablation using minimally invasive surgical techniques. J Am Coll Cardiol. 2002;40:475-80.

To assure fairness to authors submitting work for consideration in The Journal of Thoracic and Cardiovascular Surgery, a mechanism exists for managing conflicts of interest. The editor and each of the section editors complete a "Conflict of Interest" form that identifies any and all relationships with commercial and other academic entities. When the editor has a potential conflict because of a relationship with another entity or author, the editor appoints an alternate editor from among the section editors or editorial board members who assumes the entire responsibility for final decisions on the manuscript in question. The editor does not read the reviews that are submitted nor engage in discussing the manuscript prior to the final decision. When the conflict of interest involves a section editor, a "guest section editor" is appointed who fills the role normally played by the conflicted section editor. All members of the editorial board and reviewers are asked to indicate any conflict of interest when they agree to review a manuscript. 